\title{
On the Artistic Expression of Children's Picture Books
}

\author{
Yu Lei ${ }^{1, a}$ Gang Hong ${ }^{2, b}$ Lu Liu $^{3, c}$
}

\author{
${ }^{1}$ Chongqing Academy of construction science and technology, Shapingba, Chongqing, China \\ ${ }^{2}$ Chongqing RGB Animation Co., Ltd., Shapingba, Chongqing, China \\ ${ }^{3}$ Chongqing Academy of construction science and technology, Shapingba, Chongqing, China \\ a15823909717@163.com \\ b77845145@qq.com \\ c103553646@qq.com
}

\begin{abstract}
Based on the meaning of Children's picture books and combined with children's aesthetic psychology, this paper summarizes the main forms of artistic expression, that is, form abstraction, role animalization, scene planarization and good use of strong contrast color. Combined with the development of children's cognition, psychology and aesthetics, the paper sums up the three functions of children's picture books: to continue to write childlike innocence, to transmit positive energy and to stimulate the sense of pleasure.
\end{abstract}

Keywords: child plotter, art performance form, art performance function, child aesthetic psychology

\section{论儿童绘本的艺术表现}

$$
\text { 雷雨 }{ }^{1,} \text { a } \text { 洪刚 }^{2,} \text { b 刘璐 }{ }^{3,} \text { c }
$$

${ }^{l}$ 重庆建筑科技职业学院, 沙坪坝, 重庆, 中国

重庆阿尔基比动画有限公司, 沙坪坝, 重庆, 中国

${ }^{3}$ 重庆建筑科技职业学院, 沙坪坝, 重庆, 中国

a15823909717@163.com

b77845145@qq.com

c103553646@qq.com

摘要: 本文以儿童绘本意义为基础, 结合儿童审美心理, 概括其主要艺术表现形式, 即形态抽象化、角色动 物化、场景平面化、善用强对比色。并结合儿童认知、心理、审美的发展总结儿童绘本的艺术表现三大功能: 续写童真, 传递正面能量, 激发愉悦感。

关键词: 儿童绘本; 艺术表现形式; 艺术表现功能; 儿童审美心理

\section{1.儿童绘本的意义}

儿童绘本是适用于 2-6 岁儿童阅读的书籍, 其 主要的表现形式以绘画为主且带有少量文字叙述。它 是艺术作者专门针对儿童所创作的并能被广大儿童 所接受的一种绘画艺术表现形式。

在儿童教育中, 儿童绘本有着特殊的育人功能, 其对于识字阶段的儿童或艺术审美刚萌芽的幼儿有 着“以图为文”传递信息的作用。例如通过绘画的方式 传达书中文字描写的某种场景、动作、生活等, 这就 要求与之相应的儿童绘本能通过具体或抽象的绘画
形式吸引儿童及幼儿的阅读, 并能产生正确的三观引 导。

\section{2. 儿童绘本的艺术表现形式}

\section{1.意识形态抽象化}

在 2-6 岁年龄层次的幼儿绘画作品分析中发现, 该年龄层次的儿童往往是通过最简单的几何线条或 色块表现场景、景物、人物、动物、植物等。绘画与 想象的结合是他们叙述作品的主要表现方式。

因此根据此年龄阶段儿童绘画表现特征，该阶段 
的儿童读物普遍以绘本为主。根据其绘画的形式，绘 本的风格更偏向于线描与色彩平涂的结合, 儿童绘本 的意识表现往往偏向于富有想象力的抽象化、拟人化 的形态。并且儿童能在此类抽象化形态中读懂绘本内 容是最不可思议的。

例如李欧·李奥尼的《小蓝和小黄》，此部作品 被载入了 20 世纪绘本史册, 获得了凯迪克大奖。整 册绘本艺术具有强烈的极简主义风格, 它将人物几何 形象化, 以点作为认知形象出现, 并以三原色为主色, 向小读者讲述外在环境对事物的影响，同时也让抽象 的意识形态有了生命力, 是绘本历史上的一次重大创 新。

\section{2. 角色动物化}

在低幼龄儿童的绘本中, 需遵循在儿童的世界中 一切本善的原则。因此以第一人称出现的主角往往以 动物居多, 为什么会选择以动物为主要形象塑造呢?

其一, 绘本中“动物们”的呆萌的形象与儿童形象 最为接近, 儿童面对此类形象时能产生较好的代入 感。例如五年前风靡全国的国内长篇动画《喜羊羊与 灰太狼》, 该动画的贺岁电影系列制造了多起动画剧 场版票房冠军。动画中高矮胖瘦的小羊形象与现实生 活中孩子们的体型与爱好十分相似。

其二，绘本中的人物形象都有其鲜明的外部特 点。在绘本的艺术创作中如果以人为原型刻画难免显 得个性不足, 因此利用拟人的手法让外形不一的动物 们进行角色扮演, 反而让儿童们能快速接受角色设 定。例如反派形象的塑造可用狼、老虎、狐狸, 憼 态的形象可用熊、猪; 聪明的形象可用有猴子, 小猫, 小马, 小白兔。

其三, 动物的生活远离人群, 根据儿童本位论 (theory of children as standard of education) 中提出人 的天性是善良。对于动物形象的加工更加体现纯洁与 和善, 就算是在反派形象的塑造上也并非是冷酷无 情, 更多是滑稽可爱。例如, 马里奥.哈默斯的“大野 狼三部曲”, 在大野狼塑造上并没有绝对的好坏之分, 而是通过待人接物的方式去丰富 “大野狼”的人物性 格。

综上所述, 跟成人的想象力做对比, 儿童的想象 中的世界可能是远离真实世界的存在。因此, 在动物 的拟人化形象塑造时, 常用夸张的手法, 将动物的五 官特征放大。例如郑渊洁《十二生肖》绘本中小兔子 占据脸三分之一的大眼睛, 垂在肩膀的长耳朵, 作者 通过眼睛与耳朵的变化表达小兔子的情绪转变。

\section{3. 场景平面化}

对于场景的绘画方式的选择, 儿童绘本更趋向于 平面化的处理。例如, 在表现角色行走的场景中以一 条直线表达平地, 线条向上起伏表达上坡, 线条向下
延伸表达下坡。儿童绘本往往通过这种方法拉开与夸 张造型人物之间的前后关系，制造整体空间感。

对于儿童心理空间发展本身就是从二维平面到 立体空间的转换。例如儿童对于房屋的认识, 无论是 在真实空间的房屋, 还是绘本中的房屋, 儿童都能轻 松识别, 这点说明儿童已经有了空间联想认知。在“从 二维平面到立体感一儿童对于空间的心理需求研究” 一文中，作者孙建江提出“儿童虽然可以分辨对象之 间的区别，但在绘画的过程中，儿童的注意力却总是 集中在某些具有类性质的比较明显的特征上。最后画 出来的画往往缺乏细节的差异, 却具有某种间接性和 暗示。”可见, 儿童对于图像的认知往往趋向于简洁 明了化, 过于复杂的、真实的透视角度, 将画面的组 合复杂化, 不利于儿童在绘本中的快速识图, 同时也 弱化了绘“平铺直叙”的表达方式。

因此，利用儿童绘画时对于场景的表现方法，针 对绘本中房屋的表现方式，常常会选择有窗户、门、 烟图、小路等的建筑立面, 通过房屋明显的结构构件 与周围环境来表现建筑物。而这种场景平面化表现方 法正贴合儿童空间发展的认识规律。

\section{4. 善用强对比色}

对比色指在 24 色相环上相距 120 度到 180 度之 间的两种颜色。如红与绿、黄与蓝、黑与白、蓝与橙、 黄与紫等。

在美国儿科协会育儿百科中阐述“儿童视觉成长 过程中 0-3 个月的宝宝已经对鲜艳的色彩、强烈的黑 白对比感兴趣, 色彩对于这个年龄段的宝宝而言, 可 以说是有一种难以抗拒的吸引力。随着宝宝视觉系统 的发育成熟, 到了 4 个月左右, 宝宝对色彩就有了感 受能力, 可以通过认识色彩、感知色彩, 来享受美的 世界。”

同时, 根据华东师范大学的一份调查资料表明 “在诸色彩中, 儿童对于红色 (也即使太阳的颜色) 的使用最早也最为普遍。一岁使用红色为 $30.8 \%$, 二 岁使用红色为 $89.3 \%$, 占诸色的使用之冠。”

通过以上结论可见, 儿童的视觉成长有一定的周 期性, 其发展规律：红一一黑-一白一一绿- 黄——蓝——紫——灰——棕(褐色)。因此在儿童绘 本中强对比色的运用比低保度的色彩更容易激发儿 童的阅读的欲望, 其中较为出名是来自日本的著名绘 本作家宫西达也。在宫西达也的系列作品中, 作者善 用色彩浓重的画面, 并采用了极具现代风格的绘画技 法来表现人物。画面构图以角色为中心, 角色形象一 般占据画面百分之六十以上, 大开大合的画面处理让 视觉富有强烈的冲击力, 更为巧妙是在如此浓烈的画 面里阐述的却是温暖的题材。例如在绘本《你看起来 好像很好吃》中的故事画面中, 以绿色、大红、明黄 组合成强现代主义的霸王龙形象, 在白黑为主的背景 烘托下, 将高大威猛体现的淋淋尽致。此外搭配了增 
强力度感强的黑色人物轮廓线, 勾勒出一幅无比精彩 的恐龙世界。在整个绘本中文字与颜色的情感融为一 体，其色彩的新颖构思和朴实的叙事方法感染了众多 读者。

\section{3.儿童绘本的艺术表现的功能}

\section{1. 续写童真}

从以上儿童绘本的艺术表现形式可见，成年作者 对于儿童绘本的艺术创作可看作是对童真的续写，同 时也是结合自身童年生活、教育背景、生活环境的一 种真实写照。一册优秀的儿童绘本作品的创作应该体 现与之相匹配的审美取向，而此类的审美取向需尽可 能的靠近儿童所认知的世界。

这就为绘本艺术的功能表现提出了要求, 需要站 在儿童的角度出发, 了解其审美与知识接纳的实际规 律与步骤, 以图画为中心配以文字为辅助, 产生充满 奇想与创意的多彩世界。例如儿童绘本大师卡尔, 卡 尔的绘本艺术不同于其他绘本创作，他善用除了纸笔 外的拼贴方式, 是一种综合表现技法的作品呈现。 1-8 岁的儿童经历了手部动作逐步精细化的过程, 卡 尔的绘本艺术站在儿童大动作、精细动作、思维发展 的角度去感知生命的真实, 利用元素去重塑生活的趣 味, 引起儿童的共鸣, 从而达到消除他们对未知世界 恐惧的目的。

\section{2. 传递正面能量}

无论是哪种绘画形式表现的儿童绘本, 其基本出 发点在于传递正面能量。布雷顿在《儿童美术心理与 教育》里提到“当问到儿童是否喜欢一张画时, 儿童 完全按个人的喜好来决定, 即如果被描述的事物是熟 悉和都具有美好含义的, 儿童就喜欢它。”很多孩子 把画面有花、动物、家庭摆设等使他们有亲切感的事 物的画看成是美的, 而画面有恐怖暴力血色狰狞等东 西的画是丑的, 同时, 儿童通常凭感觉来选择感情丰 富的作品。因此, 利用绘本为媒介, 将原本艰深难懂 的美好文字和深刻的人生哲学, 转换为更加易于理解 的有趣画面, 儿童在阅读绘本时打破时间、空间和人 际间的隔阂, 能通过最简单的“视觉语言”达到最重要 的美育效果。

例如深受国内外儿童喜欢的绘本大师安东尼·布 朗, 在他的绘本艺术《我爸爸》中将“父爱如山”四字 以儿童的第一视角进行表现。整册故事充满夸张的想 象力, 将爸爸无所不能的“超人”形象塑造得栩栩如 生。无论是像太阳一样温暖的爸爸、能跟巨人摔跤的 爸爸、还是能轻松赶走大灰狼的爸爸, 在绘本中以一 种骄傲的口气述说出一个强壮又温柔的爸爸形象。3 岁以后的儿童开始有了对爸爸的角色认识, 这时候通 过绘本对儿童情感做出适当的引导, 能建立良好的亲 子关系, 树立父母在孩子心中的良好形象, 促进安全 感的形成。
此外， 2013 年 8 月 19 日，上海书展，进行了一 场《绘本激发正能量》的专题演讲, 整个演讲提出对 于儿童的教育可利用绘本作为传播途径对社会正能 量出力, 为培养孩子正确的审美与三观建设做好引导 作用。

\section{3. 激发愉悦感}

儿童绘本艺术常常以第一人或者第三人称为叙 述方式进行故事的展开, 儿童能结合画面快速的进入 故事情景。优秀的绘本让儿童在情景中产生愉悦感, 培养儿童积极向上, 乐观开朗的性格, 也产生了重复 阅读某册绘本的兴趣。其实这与儿童, 特别是学龄前 儿童绘本的情感设计有关。

一个优秀的绘本作家需要掌握儿童心理情绪发 展的规律与喜好的产生规律。因此, 能引导儿童行为 的绘本作品对儿童心理与生理的发展能产生积极的 作用，促进儿童在阅读时产生愉悦情绪。

在绘本中文字对儿童而言是抽象的, 但是图画、 颜色却是具象的。明亮的颜色、轻松的画面线条、强 烈的色彩对比、不同材质肌理共同组合成一系列有效 的联想画面，让儿童在阅读绘本时能直接通过画面接 收到绘本传递出的愉悦情绪。例如, 在较多儿童绘本 中, 经常运用到太阳、花朵、角色皮肤的暖色调, 如 长波系列的红色、橙色、黄色能提取出温暖、愉快之 感。经典儿童绘本《搬过来, 搬过去》就是一本以暖 色调为基调的作品, 作者在长颈鹿的皮肤、衣着、地 面、房屋、天空等颜色上都融入了长波系列的暖色调, 整个画面充满温情与愉快, 讲述着长颈鹿与鳄鱼相 遇、相知、相守的温暖故事。

\section{4. 结论}

儿童绘本艺术是以儿童审美为出发点, 以儿童心 理健康为本, 通过绘画等艺术形式对画面进行加工从 而丰富儿童对于外界事物的认知。儿童的兴趣广泛、 情感丰富、好奇心强, 所以绘本也是儿童对外界环境、 生活常识、情感引导的重要窗口。美好艺术形式可增 强绘本的表达功能, 并能更加科学有效的进行儿童绘 本创作, 更好的展示绘本艺术创作的魅力, 实现通过 绘本创作正确引导儿童达到美育的目的。

\section{REFERENCES}

[1] Sun JianJjiang,S,(1990) from two-dimensional planarity to stereopsis the study of children's psychological needs for space, Zhejiang Shi Da Xue Bao (SOCIAL SCIENCE EDITION), . J. Sci. Commun. 80-80.

[2] Steven Shelf, S, (2008) Toddler behavior, American Academy of Pediatrics Parenting Encyclopedia, Beijing Science and Technology Press, Beijing,208.

[3] Breton, (1993) Early Childhood Color, Children's 
Art Psychology and Education, Jiangsu Fine Arts Publishing House, Jiangsu,38.

[4] Zhou Jing,Z,(2007) Early Reading Development and Educational Research, Beijing Educational Science Press,Beijing.

[5] Kang Changyun,K,(2007) Study on the Reading Process of Children's Books and Story Books, Beijing Educational Science Press,Beijing. 